\title{
Neuropsychological investigation of anterior and posterior cortical function in early-stage probable Alzheimer's disease
}

\author{
P. Broks ${ }^{1}$, C. Lines², L. Atchison ${ }^{3}$, J. Challenor ${ }^{2}$, M. Traub², C. Foster ${ }^{4}$, \\ and $\mathrm{H}$. Sagar ${ }^{3}$ \\ 'School of Psychology, University of Birmingham, and Neuropsychology Unit, Royal Hallamshire \\ Hospital, Sheffield, ${ }^{2}$ Merck Sharp and Dohme Research Laboratories, Harlow, ${ }^{3}$ Department of \\ Clinical Neurology, University of Sheffield, ${ }^{4}$ Queen Mary's Hospital, Sidcup, UK
}

Correspondence to: P. Broks, School of Psychology, University of Birmingham, Edgbaston, Birmingham B15 2TT, UK

\begin{abstract}
In vivo neuroimaging studies have generally indicated a greater involvement of posterior cortical areas in early-stage dementia of the Alzheimer type (DAT) relative to frontal involvement. By contrast, some recent neuropsychological studies have shown that DAT patients perform poorly in frontal lobe tasks even in the early stages of the disease, although there is disagreement as to whether this necessarily implicates frontal pathology. The main aim of this study was to examine the hypothesis prompted by both neuroimaging studies and the traditional clinical conception of the disease, that, compared with the functioning of posterior association cortex, executive functions (thought to depend on frontal lobe integrity) are relatively spared in the early stages of DAT. A group of patients with a diagnosis of early-stage, probable DAT $(n=17)$ was compared with age- and IQ-matched controls $(n=17)$ across a range of neuropsychological tasks presumed to exercise frontal or temporoparietal functions. A profile of strengths and weaknesses was observed across 'anterior' and 'posterior' cognitive tests, including dissociations among some tests of temporoparietal function, in particular visual object perception (impaired) and spatial analysis skills (intact). Thus there was little support for the notion that the disease progresses cortically in a posterior-to-anterior direction. Possible reasons for the discrepancy between neurophysiological and neuropsychological observations are discussed, including the possibility that neuropsychological tests do not provide a valid indication of regional brain function when used in the context of DAT. Caution is urged in the clinical application of 'frontal lobe tests' for the differential diagnosis of DAT.
\end{abstract}

Keywords: Alzheimer's disease - Cortical connectivity model - Executive function - Frontal lobes - Neuropsychology

\section{INTRODUCTION}

Dementia of the Alzheimer type (DAT) is characterized by a progressive deterioration of memory and intellect. According to the prevailing clinical conception (see, for example, Huppert and Tym 1986) certain functions are prominently affected at an early stage in the disease process, such as aspects of recent memory, while others appear for some time to remain grossly intact, e.g. praxis, language functions (although it should be noted that appropriate examination may reveal signs of impairment in these areas even in the absence of symptoms). There are cases where dyspraxia, dysphasia or other focal signs are more prominent in the early stages (Shuttleworth, 1984; Moss and Albert 1988; Becker et al., 1988) but this is not the usual presentation.
Neuropathologically, DAT entails a characteristic combination of features. At the macroscopic level there is gross shrinkage of cortical gyri and ventricular enlargement, observable on routine CT scanning. Microscopic observations have shown cell loss in certain brain regions, including basal forebrain and brain stem structures, especially nucleus basalis, dorsal raphe nucleus, and locus coeruleus (Tomlinson et al., 1981; Whitehouse et al., 1981; Bondareff et al., 1982; Iversen et al, 1983; Wilcock et al, 1988). There is cell loss also in limbic structures, especially amygdala (Herzog and Kemper, 1980; Brady and Mufson, 1990) and hippocampus (Mann et al., 1985), as well as in frontal, temporal and parietal neocortical association areas (Terry et al., 1981; Najlerahim and Bowen, 
1988; Bowen et al., 1989).

In addition to these signs there are definitive histopathological changes, notably neuritic plaques (NPs) and neurofibrillary tangles (NFTs), which show a characteristic cerebral distribution. Arnold et al. (1991) observed that the regional and laminar distribution of NFTs, and to some extent NPs, suggest a pattern of cortical vulnerability determined by variations in neuronal connectivity with limbic areas. That is, the closer the connection in terms of synapses removed, the greater the vulnerability to Alzheimertype pathology. The highest concentrations of NFTs are found in medial temporal lobe structures, including the hippocampal formation, parahippocampal gyrus and amygdala. Beyond this, temporoparietal and frontal association neocortices are the most severely affected areas. Primary sensory and motor cortical regions are largely spared.

Cognitive decline in DAT is relentless and, in the later stages, the patient arrives at a state of profound incapacity apparently affecting all aspects of cognition. But, as noted above, the condition is not characterized by a uniform decline of all cognitive functions from the outset and the process of neuropsychological decline is of considerable interest. In particular, it is important to establish correlations between patterns of cognitive decline and the underlying processes of neuronal degeneration.

As noted, the limbic structures and neocortical regions typically affected in DAT show a high degree of neuronal interconnectivity, prompting the suggestion that the process of degeneration honours established neuronal pathways (Saper, 1988). It is conjectured that the disease spreads in this way from a limbic point of origin to encompass temporal, parietal and frontal association neocortices (Grady et al., 1988). Such conjecture forms the basis of the "cortical connectivity model'. As elaborated by De Lacoste and White (1993) the cortical connectivity model conceives of DAT as a 'disconnection syndrome' in so far as the subiculum and entorhinal cortex, which serve as a 'gateway' between the hippoccampus and the neocortex, are among the first structures to be affected in the disease process. In effect, this brings about a disconnection between the hippocampal formation and the rest of the brain. It is postulated that, from a limbic point of origin: (1) DAT-related neuropathology spreads in a stepwise fashion along corticocortical connections; (2) such progression correlates with the clinical severity of the disease; and (3) the involvement of different brain regions correlates with the degree of limbic-cortical interconnectivity.

There is some individual variability in the distribution of neurodegenerative changes across the neocortex but post-mortem studies are in agreement that, generally, the frontal zones are at least as heavily involved as the posterior association areas (Saper, 1988). Of course, such studies will usually involve the examination of brain specimens from individuals who have reached the end-state of the disease and there has been some suggestion that, at least in the early stages, posterior cortical areas may be more severely affected than anterior regions (Braak and Braak, 1991).

Amongst clinicians DAT has traditionally been thought to affect predominantly the posterior association areas. In support of this view in vivo analyses of cerebral metabolism and regional cerebral blood flow in DAT, using positron emission tomography (PET) and single photon emission computerized tomography (SPECT) techniques respectively, have indicated widespread abnormalities (Jagust et al., 1987; Johnson et al., 1988; Reige and Metter, 1988) but with accentuated deficits in temporoparietal regions (Haxby and Rapoport, 1986; Haxby et al., 1986; Jagust et al., 1987; Montaldi et al., 1990; Kumar et al., 1991). Such evidence of temporoparietal disorders of blood flow and metabolism in DAT has been consistent enough for some to suggest that it may constitute a hallmark of the disease (Holman, 1986).

In a review of PET studies of DAT, Rapoport (1991) observed that the consensus of both crosssectional and longitudinal studies is that the disease clearly has a predilection for the association neocortices (including frontal) as compared with the primary sensory and motor regions. However, he noted that, "Early in the disease course, this predilection is evidenced mainly in the parietal and temporal association cortices; frontal association involvement usually appears later, in more severely demented patients" (Rapoport, 1991, p.325). In terms of the cortical connectivity model this might be taken to imply that in addition to limbic-cortical stepwise progression there is a transcortical progression of the disease in a posterior to anterior direction. Or, alternatively, that the limbic-anterior advance of the disease is for some reason retarded relative to its limbic-posterior progression. If either is the case, the pattern of cognitive decline might be expected to reflect such a process with the decline of temporoparietal capacities, but a relative sparing of frontal lobe functions in the early stages.

As well as being of theoretical interest, the extent of frontal lobe involvement, if any, early in the disease process is important to consider from the viewpoint of differential diagnosis. Other forms of dementia, notably Pick's disease, have long been known to 
have a predilection for the frontal lobes, but can be difficult to distinguish from DAT on clinical grounds. More recently a different form of dementia, frontal lobe dementia (FLD), has been identified (Brun, 1987; Brun et al., 1994; Neary et al., 1988). As the name implies, FLD is characterized predominantly by pathological changes in the frontal lobes. These present as a spongiform degeneration with associated astrocytic gliosis. Histopathologically, the features of both DAT and Pick's disease are generally absent. SPECT studies have indicated frontal blood flow deficits in FLD which may distinguish the condition from DAT in vivo (Neary et al,. 1988) but diagnosis can only be confirmed histopathologically. As Bhutani et al. (1992) noted, there is as yet no reliable means of distinguishing FLD and DAT on psychometric grounds. Neary et al. (1988) reported poor performance of frontal lobe tasks by their presumed FLD patients but memory impairment was also evident and the degree of frontal lobe disorder was unrelated to disease severity. Bhutani et al. (1992) point to evidence of frontal blood flow deficits in DAT (e.g. Grady et al., 1988; Montaldi et al., 1990) which indicates frontal involvement and further complicates the differential diagnosis of FLD.

In terms of cognitive function, the evidence for frontal lobe involvement in early Alzheimer's disease is equivocal. Using tests of executive function such as the Wisconsin Card Sorting Test (WCST) some investigators have observed a relative sparing of attentional set shifting skills in early Alzheimer's disease subjects, which might be taken to reflect preserved frontal lobe function (Pillon et al., 1986; Sahakian et al., 1990). Other studies have reported evidence to suggest that frontal lobe disorder is a feature of early-stage Alzheimer's disease. Bhutani et al. (1992) investigated the performance of DAT patients, graded for disease severity, on a selection of executive tasks, including the WCST. They found marked floor effects for both DAT and normal controls on the WCST and suggest that the test is not well suited for use with elderly and dementing populations. The three other tasks employed (verbal fluency, delayed alternation, subject-ordered pointing) yielded evidence of impairment from the earliest stages of the disease. Frontal disorder may therefore be a characteristic component of early DAT, in which case, as these researchers noted, neuropsychological investigation of executive ('frontal lobe') function may not reliably distinguish DAT and FLD. The same consideration applies to Pick's disease and other degenerative diseases with frontal involvement.

Impairment of frontal lobe function in DAT has also been documented by Kessler et al. (1993). Their study also involved analysis of regional metabolic activity using PET. No correlation was found between frontal metabolic rates and performance of neuropsychological tests believed to exercise frontal functions. The authors account for the poor frontal test performance of their DAT patients in terms of generalized cognitive impairment (i.e. neuropsychologically nonspecific) and they are led to cast doubt on the validity of using 'frontal lobe tests' in the context of dementia.

The present study was designed to clarify the neuropsychological profile of early DAT using a wider range of tasks than has previously been used, including tasks widely presumed to be sensitive to anterior or posterior cortical dysfunction. In light of the evidence from in vivo neuroimaging studies that posterior cortical regions are differentially involved in early DAT, a central objective of the study was to examine the hypothesis that executive functions are relatively spared in the early stages of DAT, in the context of impaired posterior cortical function. To this end, a group of patients with a diagnosis of early-stage, probable DAT were compared with age- and IQmatched controls across a range of neuropsychological tasks classified broadly as 'anterior' or 'posterior' in terms of their presumed loading on executive function. This was done on the basis of clinical convention and/or evidence in the literature of differential anterior-posterior cerebral involvement (see Methods). Evidence of a relative sparing of executive functions would be consistent with the in vivo neurophysiological data indicating spared frontal lobe function. However, in classifying tests as either 'anterior' or 'posterior' it was recognized that, in practice, the distinction is blurred because in most cases (perhaps all) task performance will to some extent engage both anterior and posterior brain functions. No neuropsychological test gives access to a particular brain region to the exclusion of all others. (The investigation of frontal lobe functions is especially problematic in this regard and some of the conceptual pitfalls are given consideration in the Discussion.) However, in so far as neuropsychological test batteries may indicate broad patterns of function-dysfunction this method constitutes a valid initial approach to the hypothesis.

\section{METHODS}

\section{Subjects}

Patients. Seventeen community-dwelling patients ( 9 males and 8 females) judged to be suffering from mild senile DAT were included in the study. The diag- 
nosis was made according to NINCDS-ADRDA criteria for probable Alzheimer's disease (McKhann et al., 1984) on the basis of history and routine laboratory and radiological investigations (CT scanning) to exclude other possible causes of dementia such as multi-infarct dementia. Patients with symptoms or signs suggestive of a frontal-type dementia (e.g. marked changes in personality/social behaviour or prominent dysphasia) were also excluded. The judgement of severity was based on the criteria of the Clinical Dementia Rating (Berg, 1984) for mild dementia and score on the Mini-Mental State Examination (MMSE - Folstein et al., 1975).

Normal elderly. Seventeen normal age-matched controls (9 males and 8 females) from the community with no known relevant medical disorder were selected to take part in the study.

Table I shows the mean age, premorbid or current IQ (as estimated by the National Adult Reading Test [NART] - Nelson and Willison, 1991), and MMSE scores for the mild DAT patients and normal elderly. There were no significant age or IQ differences between the two groups. Vision and hearing were adequate (when corrected if necessary) in all subjects.

TABLE I. Demographic details

\begin{tabular}{lcc}
\hline & Normal elderly & Mild DAT patients \\
\hline Age & $71(60-83)^{1}$ & $73(64-83)$ \\
NART IO & $108(98-122)$ & $107(84-119)$ \\
MMSE & $28(27-30)$ & $23(18-25)$ \\
\hline
\end{tabular}

'Mean and range.

\section{Procedure}

For most subjects, tests were administered over two sessions (approximate duration $1.25 \mathrm{~h}$ per session) given on different days separated by a period of no more than 6 weeks. In some cases all testing was undertaken on a single day or spead over more than two sessions. Subjects were given the opportunity to rest between tests but there were no breaks during any individual procedure. The tasks administered during each test session were as follows:

(1) Session A: Silhouettes, Object Decision, Progressive Silhouettes, Verbal Free Recall and Recognition, Clock Test, Tower of London, Left/Right Orientation, Auditory Sustained Attention, Token Test.

(2) Session B: Position Discrimination, Number Location, Verbal Fluency, Luria Tests, Cognitive Estimates, Cube Copying, Stroop Test, Block Design, Proverbs.

Approximately half the subjects received the tests in the above order, while the other half had Session B first, followed by Session A. Testing was undertaken on a one-to-one basis by a psychologist or research nurse trained in neuropsychometric assessment.

\section{Test Classification}

Tests were designated as primarily 'posterior' or 'anterior' on the basis of clinical convention and/or evidence in the literature of differential involvement of anterior or posterior cerebral involvement. The procedures employed were also chosen to be acceptable to the patient population, so some standard tests which dementing patients have difficulty understanding, such as the WCST, were not included.

\section{Posterior Tests}

Verbal Free Recall and Recognition. The subject was presented with a list of 10 high frequency and imageability nouns, one at a time, on a computer screen. Presentation rates were controlled by the experimenter depending on the time taken by the subject to pronounce the word correctly, although there was a minimum presentation time of $2 \mathrm{~s}$. At the end of the presentation the subject was given a maximum of $60 \mathrm{~s}$ to recall as many words as he/she could, in any order. This procedure was repeated for four trials using the same word list presented in the same order each time. The measures analysed were the mean number of words recalled and total number of intrusions. Following the last free recall trial a three-choice recognition test was administered. For each of the original 10 words, three words (the target word and two semantically related words) were displayed on the computer screen. The subject had to indicate which word had been in the original list. The measure analyzed was the number of words correctly recognized.

Block Design. The first seven items of the WAIS-R block design test (Wechsler, 1981) were administered. This test requires the subject to manipulate coloured blocks until they match a given design, within specified time limits. Full details of administration and scoring can be found in the WAIS-R manual. The measure analyzed was the total number of points scored.

Cube Copying: The subject was provided with a 3dimensional cube drawing identical to that used in Moore and Wyke (1984) and asked to copy it. Copies were scored on a 15 point scale based on the criteria given in Moore and Wyke (1984). Each of the following items was given 1 point if correct: lines 1, 2, 3 and 
4 of the front face; lines 1, 2, 3, 4 and 5 of the top and sides; attempt to draw perspective; no extra lines present; all lines connected; no spatial distortions; approximately same size as original; approximately same orientation as original. The measure analyzed was the total score.

Clock Test. The subject was first given a blank sheet of paper and asked to draw a clock face from memory and set the hands to 11:10. Drawings were scored on an 8-point scale (circle/square present, all numbers present, numbers in correct position, numbers equally spaced, numbers correctly oriented, hands in correct position, short and long hand present and in correct relation, no additions) and the total score was analyzed. The subject was then provided with a picture of a clock face with the hands set to $11: 10$ and asked to copy it. Copies were scored on an 11-point scale (same as for clock drawing plus: arrows on hands and central pivot present, copy approximately same size as original, no overlap with original) and the total score was analyzed. Next, in the setting test, the subject was provided with pictures of nine empty clock faces (no hands) and asked to draw in hands corresponding to various times (three 'o'clock', three 'past' and three 'to' positions) written below each clock. The number correct was analyzed. Finally, for the clock reading test, the subject was shown pictures of nine clocks set to various times (three 'o' clock, three 'past' and three 'to' positions) and asked to read the time. The total number of correct responses was analysed (see Freedman et al., 1994, for a recent neuropsychological assessment of clock drawing procedures).

Left/Right Orientation. The procedure employed by Fischer et al. (1990) was followed. The subject was first asked to point to six lateral parts of their own body (left hand, right eye, left ear, right shoulder, right hand, left knee). A toy doll was then placed opposite the subject and they were required to point to six parts of the doll's body (the doll's left ear, right shoulder, left knee, right eye, left hand, right ear). The number of correct responses was analyzed.

Token Test. Ten coloured plastic circles and squares were placed in two rows on a table in front of the subject (top row: blue, white, green, yellow and red circles; bottom row: green, yellow, red, blue and white squares). The subject was then asked to perform 13 commands from Part 5 of the original Token Test (i.e. the most difficult items, the items used were those incorporated in DeRenzi and Faglioni's (1978) Short
Version of the Token Test), e.g. 'put the red circle between the yellow square and the green square'. Following each command the tokens were returned to their original position. The measure analyzed was the total number of correct responses.

Visual Object and Space Perception Battery (VOSP). Full details concerning the VOSP tests can be found in the test manual (Warrington and James, 1991). The following are brief descriptions of each test.

Silhouettes. In part A of the test the subject was presented with silhouette drawings of 15 animals and asked to name them. Part B consisted of 15 silhouette drawings of common objects. The measure analyzed was the number of correct responses.

Object Decision. The subject was presented with 20 arrays, each consisting of a silhouette drawing of a real object along with three nonsense shapes. The subject was required to point to the real object. The number of correct responses was analyzed.

Progressive Silhouettes. The subject was presented with a sequence of a maximum of ten silhouette drawings of the same familiar object viewed from unusual angles. As each drawing was shown the object became progressively easier to identify. The score was the number of pictures that it was necessary to show the subject before he/she identified the object. The procedure was repeated for a second object. The measure analyzed was the total number of trials required to name the two objects.

Position Discrimination. The subject was presented with 20 test stimuli each consisting of two adjacent horizontal squares, one with a black dot printed exactly in the centre and one with a black dot just off centre. The subject was asked to indicate in which box the central dot lay. The measure analyzed was the number of correct responses.

Number Location. The subject was presented with 10 test stimuli each consisting of two squares one above the other. The top square contained randomly placed numbers and in the bottom square a single black dot was placed in a position corresponding to one of the numbers. The subject was asked to identify which number in the top square was in the same position as the dot in the bottom square. The measure analyzed was the number of correct responses. 


\section{Anterior Tests}

Auditory Sustained Attention. This test was developed from a procedure used by Wilkins et al. (1987). The subject was required to listen to trains of computer-generated beeps. At the end of each train the subject was asked to tell the experimenter the number of beeps they had heard. Ten trains, varying in length from 5-14 beeps, were presented. The beeps in each train had a duration of 50 milliseconds and were separated by a randomly chosen period of 2-6 s. A practice train was given prior to the start of testing. The measure analyzed was the number of correct responses.

Tower of London. This test was based on the procedure described by Shallice (1982). The apparatus consisted of three vertical metal poles set in a line on a plastic base, plus three coloured (red, white and green) plastic disks with a hole drilled in the middle so that they could be placed on the metal poles. The subject was given one set of apparatus while the experimenter used an identical piece. At the beginning of the test the subject's disks were placed in the following positions: red disk on top of the green disk on the first pole, white disk on the second pole, third pole empty. The experimenter's disks were then placed as follows: first pole empty, red disk on top of the white disk on the second pole, green disk on the third pole. The experimenter then explained to the subject that the task was to move the disks on their set of poles so that they ended up in the same positions as on the experimenter's apparatus and that they should do this in the fewest number of moves possible. The experimenter then demonstrated the solution to the first problem and the subject was allowed to practice until they could solve this problem in the minimum number of moves (2). When the experimenter was satisfied that the subject understood the nature of the task they were given six further problems of increasing difficulty to solve (minimum number of moves: 2, 3, 3, 4, 4, 5 respectively). The starting position of disks on the subject's apparatus was always the same. If the subject did not solve the problem in the minimum numbers of moves on the first trial he/she was given a second trial. Testing was abandoned if the subject was unable to solve a problem. The measures analyzed were the number of problems solved in the minimum number of moves on trial 1 , and the number of further problems solved in the minimum number of moves.

Verbal Fluency. The subject was given 1 min to name as many animals as he/she could think of. The procedure was repeated for words beginning with the let- ters ' $\mathrm{S}$ ' and ' $\mathrm{J}$ ', excluding names. The measure analyzed was the number of correct words excluding repetitions.

Luria Tests. Two procedures were taken from Christensen's modification of Luria's neuropsychological investigation (Christensen, 1974) and further modified to allow quantitative analysis of performance. For the manual sequencing test the experimenter demonstrated a sequence of hand movements, using one hand only - fist on table, palm on table, side of hand on table. The subject copied these movements until the experimenter was satisfied that he/she understood the task. The subject was then required to perform as many correct sequences as possible in $30 \mathrm{~s}$. The total number of correct sequences was analyzed. For the manual alternation test the subject was required to switch between the following hand positions using alternate hands - fist on table with one hand, palm on table with the other. As for the sequencing test the procedure was demonstrated until the experimenter was satisfied that the subject understood it. The subject was then given $30 \mathrm{~s}$ to perform as many alternations as possible. The number of correct alternations was analyzed.

Cognitive Estimates. The subject was asked eight questions which he/she was unlikely to know the answers to and hence would have to solve by having an 'educated guess'. The following questions from Shallice and Evans (1978), all of which have a numeric answer, were asked: "How fast do race horses gallop?; What is the height of the tallest building in Britain?; What is the length of an average man's spine?; What is the age of the oldest person in Britain?; How tall is the average English woman?; How heavy is a full pint bottle of milk?; What is the width of a double decker bus?; How many camels are there in Holland?" The measure analyzed was the number of 'reasonable' answers. For each question a reasonable answer was defined as one lying within 1 standard deviation of the mean value for the normal elderly subjects.

Stroop Test. A commercially available Stroop test was used (Trenerry et al., 1989, see test manual for full details). The subject was first asked to read 112 colour names from a sheet containing colour words written in non-matching colours (control/word reading condition). Next, in the interference/ink colour naming condition, the subject was asked to name the colour of the ink that the work was written in. The subject was allowed $2 \mathrm{~min}$ for each part of the test. 
The measure analyzed was the number of correct responses.

\section{Data Analysis}

Data were analysed either by $t$-tests for independent samples or, if the data were not normally distributed or did not conform to interval scaling, by Mann-Whitney non-parametric tests to determine whether the mild DAT group differed from the normal elderly. The analysis was undertaken using the BMDP program $3 \mathrm{D}$. In view of the relatively large number of comparisons being made, and in order to identify measures on which DAT patients were clearly impaired, the level of significance was set at $p<0.01$. Complete data were available for all tests except the following: clock drawing (normal elderly $n=15$, mild DAT $n=16$ ); clock copying (normal elderly $n=16$, mild DAT $n=15$ ); cube copying (normal elderly $n=16$, mild DAT $n=15$ ).

\section{RESULTS}

The means and standard deviations for each measure are given in Tables II (posterior tests) and III (frontal tests). The main features of the results are outlined below and summarised in Table IV.

\section{Posterior Tests}

Verbal Free Recall and Recognition. As expected, the mild DAT patients performed poorly on this test and were significantly worse than the normal elderly subjects $(p=0.0000)$. The patients also exhibited a statistically significant impairment in recognizing previously seen words compared to the normal elderly $(p=0.005)$, even though they actually performed the task quite well (90\% accuracy) compared to the free recall test. The difference in the number of non-list intrusions was not significant $(p=0.03)$.

Block Design. As anticipated, the mild DAT patients performed significantly worse than the normal elderly subjects on this task $(p=0.0001)$.

Cube Copying. The normal elderly subjects performed surprisingly poorly on this test and the mild DAT patients were not significantly worse $(p=0.3)$.

Clock Test. The mild DAT group were significantly impaired at clock drawing $(p=0.0001)$ and clock reading $(p=0.0007)$, but not at clock copying $(p=0.02)$ and setting $(p=0.01)$.
Left/Right Orientation. Both the mild DAT patients and normal elderly subjects performed the task well and there were no significant differences between group differences for self $(p=0.3)$ or opposing doll $(p=0.03)$ orientation.

Token Test. The test was performed well by both groups of subjects and there was no significant difference in scores $(p=0.04)$.

VOSP Battery. Compared with the normal elderly subjects, the mild DAT patients were significantly impaired on the silhouettes A $(p=0.0003)$ and $\mathrm{B}$ $(p=0.0001)$ and object decision $(p=0.0002)$ tests but not on the progressive silhouettes $(p=0.04)$, position discrimination $(p=0.2)$ and number location $(p=0.2)$ tasks.

\section{Executive Tests}

Auditory Sustained Attention. Despite being placed at the end of a test session, when subjects would have been tired, this task was performed well by both the

TABLE II. Posterior test results

\begin{tabular}{|c|c|c|c|c|}
\hline Test & Measure & Range & $\begin{array}{l}\text { Normal } \\
\text { elderly }\end{array}$ & $\begin{array}{l}\text { Mild DAT } \\
\text { patients }\end{array}$ \\
\hline \multirow{3}{*}{$\begin{array}{l}\text { Verbal Free } \\
\text { Recall }\end{array}$} & Recall & $0-10$ & $7.7(1.0)^{1}$ & $4.1(1.1)^{*}$ \\
\hline & Intrusions $^{2}$ & & $0.2(0.5)$ & $1.0(1.6)$ \\
\hline & & $0-10$ & $9.8(0.7)$ & $8.9(1.5)^{*}$ \\
\hline \multicolumn{2}{|l|}{ Block design } & $0-37$ & $23.8(4.0)$ & $12.4(8.3)^{*}$ \\
\hline \multicolumn{2}{|c|}{ Cube Copying } & $0-15$ & $10.4(3.0)$ & $8.9(4.2)$ \\
\hline \multirow[t]{4}{*}{ Clock } & Drawing & 0.8 & $6.9(1.2)$ & $3.9(2.2)^{*}$ \\
\hline & Copying & $0-11$ & $10.3(1.3)$ & $8.9(1.6)$ \\
\hline & Setting & $0-9$ & $8.5(0.9)$ & $6.6(2.4)$ \\
\hline & Reading & $0-9$ & $9.0(0.0)$ & $7.5(2.1)^{*}$ \\
\hline Left/Right & $\begin{array}{l}\text { Self } \\
\text { Opposing doll }\end{array}$ & $\begin{array}{l}0-6 \\
0-6\end{array}$ & $\begin{array}{l}6.0(0.0) \\
5.9(0.2)\end{array}$ & $\begin{array}{l}5.9(0.2) \\
5.1(1.7)\end{array}$ \\
\hline \multicolumn{2}{|l|}{ Token } & $0-13$ & $12.3(1.3)$ & $11.1(1.8)$ \\
\hline \multirow[t]{6}{*}{ VOSP } & Silhouettes A & $0-15$ & $9.9(2.5)$ & $6.4(2.6)^{*}$ \\
\hline & Silhouettes B & $0-15$ & $11.1(2.0)$ & $7.5(2.6)^{*}$ \\
\hline & $\begin{array}{l}\text { Progressive } \\
\text { silhouettes }^{2}\end{array}$ & $2-20$ & $11.5(2.5)$ & $13.3(2.1)$ \\
\hline & $\begin{array}{l}\text { Object } \\
\text { decision }\end{array}$ & $0-20$ & $17.9(2.3)$ & $14.8(2.0)^{*}$ \\
\hline & $\begin{array}{l}\text { Position } \\
\text { discrimination }\end{array}$ & $n^{0-20}$ & $19.5(0.9)$ & $19.1(1.1)$ \\
\hline & $\begin{array}{l}\text { Number } \\
\text { location }\end{array}$ & $0-10$ & $9.2(1.0)$ & $8.0(2.3)$ \\
\hline
\end{tabular}

${ }^{1}$ Mean and standard deviation.

${ }^{2}$ For these measures a lower score is better. For all other measures a higher score is better.

${ }^{*} p<0.01$, significantly worse than normal elderly subjects. 
normal elderly subjects and the mild DAT patients and there was no significant difference between groups $(p=0.3)$.

Tower of London. The mild DAT patients were significantly worse than the normal elderly subjects at solving problems in the minimum numbers of moves on trial $1(p=0.002)$. However, given the apparent complexity of the task, their performance was better than anticipated and this is reflected in the finding that there was no significant difference between groups in the number of problems solved in the minimum number of moves when subjects were allowed two attempts $(p=0.02)$.

Verbal Fluency. As expected the mild DAT patients were significantly impaired on tests of category (animals) and letter ('S') fluency ( $p=0.0001$ and 0.009 respectively). Both groups could only recall a few words beginning with the letter ' $\mathrm{J}$ ' and did not differ significantly on this measure $(p=0.1)$.

Luria Tests. There was no statistically significant difference between groups on the sequencing measure $(p=0.1)$. However, the mild DAT patients were significantly worse than the normal elderly subjects on the manual alternation task $(p=0.009)$.

Cognitive Estimates. Both groups of subjects produced reasonable answers to the questions and there was no significant difference between groups $(p=0.01)$.

Stroop Test. There was no difference between the groups on the word reading (control) condition ( $p$ $=0.1$ ). However, when the task was changed to reading the ink colour (interference condition) the mild
TABLE III. Executive test results

\begin{tabular}{lllll}
\hline Test & Measure & Range & $\begin{array}{l}\text { Normal } \\
\text { elderly }\end{array}$ & $\begin{array}{l}\text { Mild DAT } \\
\text { patients }\end{array}$ \\
\hline $\begin{array}{c}\text { Auditory } \\
\text { attention }\end{array}$ & & $0-10$ & $9.6(1.0)^{1}$ & $9.2(1.1)$ \\
$\begin{array}{c}\text { Tower of } \\
\text { London }\end{array}$ & Correct Trial 1 & $0-6$ & $5.4(0.9)$ & $4.0(1.3)^{*}$ \\
& $\begin{array}{c}\text { Correct in } \\
\text { Minimum }\end{array}$ & $0-6$ & $5.8(0.4)$ & $5.1(1.1)$ \\
& Animals & & $18.8(4.0)$ & $11.9(4.0)^{*}$ \\
$\begin{array}{c}\text { Verbal } \\
\text { Fluency }\end{array}$ & S & & $13.5(6.5)$ & $8.4(4.0)^{*}$ \\
& $\mathrm{~J}$ & & $6.9(2.8)$ & $5.1(3.8)$ \\
Luria & Sequencing & & $14.7(4.4)$ & $11.5(7.5)$ \\
& Alternations & & $40.8(18.5)$ & $23.5(17.9)^{*}$ \\
Cognitive & & $0-8$ & $6.4(1.3)$ & $5.0(1.3)$ \\
Estimates & & & & \\
Stroop & $\begin{array}{l}\text { Word Reading } \\
\text { Ink Reading }\end{array}$ & $0-112$ & $111.9(0.2)$ & $111.1(1.3)$ \\
& $0-112$ & $74.5(25.4)$ & $34.1(19.2)^{*}$ \\
\hline
\end{tabular}

For all measures a higher score is better.

'Mean and standard deviation.

${ }^{*} p<0.01$, significantly worse than normal elderly subjects.

DAT patients exhibited a greater decrement in performance than the normal elderly subject $(p=0.0000)$.

\section{Summary of test results}

Table IV classifies the tests according to whether the DAT group did or did not show significant impairment relative to controls.

\section{DISCUSSION}

A profile of strengths and weaknesses in the neuropsychological test performance of the DAT subjects emerged. Marked cognitive deficits were evident

TABLE IV. Classification of test results into measures showing minimal, or no impairment, or significant impairment in mild DAT patients relative to normal elderly controls

\begin{tabular}{|c|c|}
\hline Minimal or no impairment ${ }^{1}$ & Significant impairment $^{2}$ \\
\hline $\begin{array}{l}\text { Verbal free recall: intrusions } \\
\text { Cube copying } \\
\text { Clock copying and setting } \\
\text { Left/right orientation: self, opposing doll } \\
\text { Token test } \\
\text { VOSP: progressive silhouettes, position discrimination, number location } \\
\text { Auditory sustained attention } \\
\text { Tower of London: correct in minimum number of moves } \\
\text { Luria: sequencing } \\
\text { Cognitive estimates } \\
\text { Stroop: word reading }\end{array}$ & $\begin{array}{l}\text { Verbal free recall and recognition } \\
\text { Block design } \\
\text { Clock drawing and reading } \\
\text { VOSP: silhouettes ( } A \text { and } B \text { ), object } \\
\text { decision } \\
\text { Tower of London: correct in minimum } \\
\text { number of moves on trial } 1 \\
\text { Verbal fluency (except ' } \mathrm{J} \text { ') } \\
\text { Luria: alternations } \\
\text { Stroop: ink readings }\end{array}$ \\
\hline
\end{tabular}

${ }^{1} p>0.01$

${ }^{2} p<0.01$

142 Behavioural Neurology • Vol 9 . 1996 
across a range of tasks including, predictably, tests of new learning (verbal free recall) and constructional ability (block design; clock drawing). Such deficits are well established as features of early DAT (Huppert and Tym, 1986). Yet in somewhat over half of the tasks administered the performance of the DAT group was comparable to that of the controls, suggesting that poor performance on the other tests cannot be simply attributed to generalized cognitive impairment.

The pattern of results thus appears at first sight not to support the suggestion prompted by evidence from in vivo neuroimaging studies (see Rapoport, 1991 for a review) that executive functions (conventionally understood to depend on the integrity of the frontal lobes) may be spared in the early stages of DAT relative to temporoparietal functioning. A majority of the tasks considered to be 'frontal sensitive' in fact revealed significant deficits in the DAT group, including tests of planning/problem solving, verbal-cognitive fluency, selective attention, motor executive function (Tower of London, Verbal Fluency, Stroop test, Luria manual alternation task). At the same time performance of certain other tests considered to exercise more posterior cortical functions was unimpaired - for example, Left/Right orientation, Visuospatial Perception (VOSP), verbal comprehension (Token Test). To the extent that frontal lobe dysfunction is implicated, our results would support and extend the observations of Bhutani et al. (1992) who also found evidence of executive dysfunction in the early stages of DAT. On this interpretation, the obtained pattern of results might be taken to suggest that the in vivo neurophysiological evidence is misleading in not identifying anterior cortical dysfunction in the early stages of DAT. However, the apparent discrepancy between the present neuropsychological findings and the available neurophysiological evidence is open to other interpretations. As an alternative, it might be argued that the approach to neuropsychological testing used in this and related studies is inadequate for the purposes of charting brain-behaviour correlations in DAT, in so far as there may be doubts about the validity of the tests as indicators of regional brain dysfunction. Each of these opposing perspectives will be considered before turning to other observations.

If the present results are taken at face value to indicate early frontal lobe involvement in DAT how might such an observation be reconciled with the evidence from in vivo neurophysiological studies which suggest a relative lack of early frontal involvement? For example, Rapoport (1991) in his review of PET scan studies makes the point that frontal involvement usually only appears in the later stages when patients are more severely demented. It cannot easily be argued that the subjects of the present study were at an advanced stage of the disease (and therefore more likely to show global cortical involvement). In so far as subject selection criteria are comparable across the various studies, it would be generally accepted that our subjects represent 'mild' or early stage DAT.

It might be the case that neuropsychological methods are more sensitive in detecting disease-related functional decline. The neurophysiological methods record physical brain activities in the form of blood flow or metabolic data which, reasonably enough, are presumed to correlate with higher-order brain function, whereas the neuropsychological approach (by definition) accesses higher-order brain function directly. A problem arises for the interpretation of neurophysiological data if the correlation between discernible changes in physical brain state and cognitive status is uncertain or unreliable. It may be that disorder of frontal lobe functioning occurs in the early stages of DAT without initially being associated with detectable changes at the gross physiological level (but perhaps anticipating such physiological changes). In this scenario the observed physiological changes are conceived as being relatively loosely related to clinically (and neuropsychologically) apparent changes in functional capacity. This sort of interpretation may be plausible in terms of accounting for discrepancies between neuropsychological and neurophysiological data (the different methodologies may simply be probing different aspects of disease progression which, as suggested, may have relatively loose linkage with one another) and it clearly emphasizes the need for caution in cross-referencing descriptions of disease progression at different levels of analysis. However, it begs the question of why physiological changes should be more apparent in posterior regions of the brain in the early stages of the disease if not due to differential involvement of those regions.

Another view which might accommodate the two forms of evidence is that the neuropsychological signs of frontal lobe disorder are a secondary effect of temporal lobe disease rather than a primary consequence of frontal lesions. The concept of secondary dysfunctions in areas remote from lesion sites ('diaschisis') has a long history in behavioural neurology (von Monakow, 1911/1960). In the present case, for example, it may be relevant to note that the amygdala appears to be involved early in the disease process (Mann, 1992) and there are known to be rich recip- 
rocal connections between the amygdala and the frontal lobes (Amaral et al., 1992) which could, conceivably, provide a basis for distal effects. Thus disease of the amygdala might impair the functional integrity of the frontal lobes without there being any primary pathology of the frontal cortex, analogous to the way in which frontal-type cognitive dysfunction may be observed in Parkinson's disease as a secondary consequence of basal ganglia disease (Owen et al., 1992). There is in fact some evidence that restricted bilateral amygdala lesions may lead to executive dysfunction (Tranel and Hyman, 1990). Against this interpretation, there is evidence that, at least in some cases, damage at one location may produce changes detectable by PET scanning in other, structurally intact, areas (e.g. Pappata et al., 1990). The generally negative findings of PET studies would tend to suggest that the frontal lobes are not affected in this way in DAT. This in itself would seem to call for explanation given the massive connectivity between anterior temporal and frontal zones and the well-established early pathology of the temporal lobes in DAT. But it should be noted that physiological evidence for diaschisis between ipsilateral cortical areas is generally weaker than for subcorticalcortical and transcallosal routes. Thus, for example, although it is widely accepted that intrahemispheric diaschisis may contribute to clinical deficits such as stroke-associated aphasia, the physiological evidence for such effects remains unconvincing (Soh et al., 1978; Samson et al., 1985). No study has yet examined for effects on frontal lobe physiology in cases of focal damage to temporal structures such as the amygdala and, in general, detailed knowledge of the mechanisms responsible for remote physiological effects between damaged and intact structures in different brain regions is lacking. Explanations of the present results in terms of diaschisis therefore remain speculative.

With regard to possible limitations of the neuropsychological methodology there are two general issues to consider: the first concerns the construct validity of the neuropsychological tests employed, i.e. the matter of whether or not they sample the regional brain functions they purport to; secondly, there is the question of differential test sensitivity, i.e. the potentially confounding effects of neuropsychologically non-specific variations in test difficulty. These two issues are related in that variations in sensitivity ('difficulty') would complicate the interpretation of test results in terms of underlying patterns of brain (dys)function. In the context of the present study, there might be some systematic bias favouring either anterior or posterior tests en bloc which, in the latter case, would lead to Type 1 errors of interpretation. Alternatively, the variation might be random across 'anterior' and 'posterior' domains of function, tending to obscure true neuropsychological patterns and disposing toward Type 2 error.

The problem of differential test sensitivity is common to all studies seeking to draw inferences about brain-behaviour relationships on the basis of neuropsychological test profiles. It stems from the fact that there is no sure way of equating tests for difficulty. The DAT patients showed variable test performance within and across the 'anterior/posterior' sub-batteries (see Table IV) and, intuitively at least, there seems little to indicate that the pattern of results is determined by the apparent 'difficulty' of the tasks. In some cases the obtained results seem counterintuitive in this regard. For example, the DAT patients had no difficulty with the auditory sustained attention test relative to controls and yet this is a task generally experienced as fairly demanding by normal subjects. A tone counting task, it requires a sustained effort of concentration over a period of around 12 min. By contrast they performed poorly in the Luria manual alternation task, which is not generally experienced as demanding by normal subjects. While not without interest, subjective accounts of test difficulty are of limited value in this context. The problem of differential test sensitivity can only be overcome convincingly by the demonstration of 'double dissociations' between patients and task performance (Shallice, 1988). It can also be addressed to some extent by examining the specificity of the cognitive profile observed in DAT (without necessarily demonstrating clear double dissociations of function). If variations in cognitive profile are observed for other forms of pathology, in terms of patterns of single dissociation, then such variations could be inferred to have some neuropsychological significance beyond any trivial 'test sensitivity' effects. It is acknowledged that any inferences drawn from the present study regarding brain-behaviour patterns would be strengthened by the demonstration of variant profiles in other patient groups. Our preliminary investigations of patients with Parkinson's disease and with depressed patients using tasks similar to some of those employed in the present study indicate that these groups may indeed show variations in cognitive profile uncharacteristic of DAT and we are seeking confirmation of these observations.

The problem of test construct validity is also a feature of all studies adopting the neuropsychological test battery approach. In recognition of this problem 
the present study involved the use of a wide range of procedures whose validity derives from previous application in experimental studies and/or widespread use in clinical neuropsychological practice. This might not be sufficient, however, because the validity of certain tests may vary according to the populations in which they are applied. For example, it is arguable that in cases where there is presumed nonfrontal pathology (such as DAT) impaired performance of executive tasks might be a secondary consequence of more general impairment of instrumental functions, such as language and praxis, rather than primary functions of the frontal lobes such as response initiation or strategic planning. Such primary abilities cannot be examined in isolation from the instrumental functions through which they are expressed. Had there been a clear dissociation between impaired posterior and intact anterior test performance then it would have been possible to claim conformity with in vivo neurophysiological studies in line with the hypothesis that anterior brain functions are relatively spared in early DAT. In light of the more complex pattern of results that actually emerged, we can only observe that our data do not conform with the neurophysiological evidence. For the reasons given, we cannot conclude with confidence that this lack of conformity implies patterns of brain dysfunction at odds with the neurophysiological evidence. That is, we are unable to conclude that executive functions per se are disrupted, merely that our DAT patients performed poorly in some tests which are commonly used to assess executive function. It remains possible that posterior brain dysfunction may have contributed to impairment in some of the executive tasks. Future studies might usefully attempt to deconstruct tasks in terms of the extent to which their performance depends upon instrumental functions which are not frontally mediated. In this regard, one might argue that the efficient performance of the DAT patients in the auditory vigilance task provides evidence of frontal sparing. Although in other ways demanding, this task apparently places little load on 'posterior' functions in that there are minimal demands on perception (listening to pure tones) and it requires a simple vocal response (utterance of a one or two digit number). By contrast, tests such as the Tower of London task, which the DAT patients performed less well, are relatively complex perceptually and motorically. Again, it should also be borne in mind that the frontal lobes are anatomically complex and functionally heterogeneous and it may be that some aspects of front lobe functioning are affected in early-stage DAT, but not others.
Doubts about the relationship between frontal pathology and frontal-type cognitive disorder in DAT are also raised in a recent PET study reported by Kessler et al. (1993). They found that the poor performance of their DAT patients on frontal lobe tests correlated with reduced temporoparietal glucose metabolism but not with rates of metabolism in the frontal lobes. They were led to doubt the validity of 'frontal lobe tests' when used in the context of dementia. They suggest that poor performance in such tasks is a by-product of generalized cognitive impairment rather than specific frontal lobe disorder. However, their data could be accommodated by the notion of 'secondary' frontal dysfunction (e.g. in consequence of the effects of temporal pathology). Also, the present study shows that, contrary to the suggestion of Kessler et al. (1993), signs of possible frontal lobe dysfunction can occur in mild DAT in the context of a wider profile of preserved and impaired abilities, in which sense our patients may not be said to have 'generalized cognitive impairment'. Indeed areas of preservation and deficit were found among both 'anterior' and 'posterior' tests and it is to this observation that we now turn.

The main concern of the present study was to examine the neuropsychological profile of early DAT in broad terms, viz. anterior versus posterior functions. However, some components of the test battery yielded data of relevance to issues beyond the general question of the integrity of front lobe functioning and merit further discussion. For example, the DAT group performed at levels comparable with the controls in the two spatial analysis tests (position discrimination and number location) selected from VOSP. Their performance of these tasks was also well within normal limits according to the published norms for the tests (Warrington and James, 1991). By contrast, in two of the three VOSP object recognition tests administered (object decision and silhouettes) the performance of the DAT group was impaired relative to controls and according to the published test criteria. Dissociation between spatial analysis skills (intact) and object perception skills (impaired) in DAT has not previously been reported. Dissociations of object and space perceptions have previously been reported in connection with focal brain lesions. Both forms of perceptual disorder are generally associated with post-rolandic right hemisphere lesions, with indications that spatial disorders may be associated with more posterior damage (Newcombe and Russell, 1969; Warrington and James, 1967, 1988). Neuroanatomical studies indicate that the primate brain contains two distinct visual pathways originating 
in the striate cortex. One pathway projects to the parietal lobe and is held to convey information for spatial analysis; the other, directed towards the temporal lobes, is believed to be concerned with analysis of the properties of objects (Ungerleider and Mishkin, 1982; Maunsell and Newsome, 1987). There is some neuropsychological evidence for such a functional/neuroanatomical separation of object and space perception in humans (Newcombe and Russell, 1969; Newcombe et al., 1987, Ungerleider and Haxby, 1994). In these terms the present observations might be taken to reflect a greater involvement of temporal compared with parietal areas in early-stage DAT (consistent with the cortical connectivity model), in which case tests of visual cognition might prove to be of use in staging disease progression. However, it should be noted that deficits of both object and space perception have been linked with parietal damage alone (for review see McCarthy and Warrington, 1990). The neuropsychological basis of the observed dissociation between object and space perception in our DAT patients thus remains uncertain, but further studies to clarify the relation between visual cognitive skills and the progression of Alzheimer's disease are clearly indicated.

Another test which presented no significant difficulty for the DAT group, the auditory vigilance task, also deserves mention. A version of this task has been shown to be sensitive to frontal lobe disorder, particularly when a lesion of the right frontal lobe is involved (Wilkins et al., 1987). Also, in previous studies of the effects of the anticholinergic drug scopolamine on memory and attention in healthy volunteers we have shown the task to be highly sensitive to cholinergic blockage (Broks et al., 1988). On the basis of that evidence (and against the background of the cholinergic hypothesis of DAT) it was suggested that difficulties with the task might show early in the course of Alzheimer's disease. This notion has since been challenged by Lines et al. (1991) who found that DAT subjects did not differ from normal controls in their ability to perform the auditory vigilance task at stimulus presentation frequencies sensitive to experimental cholinergic blockade. In one respect the present study amounts to a replication of those findings and further undermines the 'scopolamine model' of DAT.

In summary, the main findings of the present study were as follows. Patients in the early stages of DAT performed poorly in various clinical and experimental tasks presumed to test executive functions. A profile of strengths and weaknesses was observed across a range of both 'anterior' and 'posterior' cognitive tests, including dissociations among some tests of temporoparietal function. Thus no support was found for the notion that the disease progresses cortically in a posterior-to-anterior fashion, and it is acknowledged that this is inconsistent with evidence obtained from in vivo neuroimaging studies. As noted above, reasons for the apparent discrepancy between the neuroimaging evidence and our own observations include the possibility that conventional neuropsychologial testing (grounded in the clinical test battery approach) may not provide a valid indication of regional brain dysfunction when used in the assessment of Alzheimer's disease. We have also considered possible psychobiological explanations of the discrepancy. Previous investigators who have documented executive dysfunction in DAT have argued either that there is primary frontal involvement in DAT at an early stage (Bhutani et al., 1992) or, alternatively, that there is no early frontal involvement and that observed 'frontal deficits' are neuropsychologically non-specific (Kessler et al., 1993). In the present paper we have considered a third possibility, that poor performance on frontal lobe tasks may be neuropsychologically specific (i.e. not merely a by-product of 'generalised cognitive impairment') but not due to primary frontal pathology. Rather it is tentatively suggested that frontal disorder may be a secondary consequence of temporal disease. Whatever the underlying pathology, poor performance on executive function tasks would appear to be a feature of the neuropsychological profile of early-stage DAT. In this light we would, along with other commentators (Bhutani et al., 1992), urge caution in the clinical application of 'frontal lobe tests' for the differentiation of DAT and dementias with a frontal lobe emphasis.

\section{Acknowledgement}

We would like to thank Professor E.K. Warrington for her helpful comments concerning the results of the visual object and space perception tests.

\section{REFERENCES}

Amaral, Price JL, Pitkänen A and Carmichael ST (1992) Anatomical organisation of the primate amygdaloid complex. In: The Amygdala: Neurobiological Aspects of Emotion, Memory, and Mental Dysfunction (Ed. JP Aggleton), Wiley, New York pp. 1-66.

Arnold SE, Hyman BT, Flory J, Damasio AR and Van Hoesen GW (1991) The topographical and neuroanatomical distribution of neurofibrillary tangles and neuritic plaques in the cerebral cortex of patients with Alzheimer's disease. Cerebral Cortex, 1, 103-116.

Becker JT, Huff FJ, Nebes RD, Holland A and Boller F (1988) Neuropsychological function in Alzheimer's disease: pattern of impairment and rates of progression. Archives of Neurology, 45, 263-268. 
Berg L (1984) Clinical dementia rating. British Journal of Psychiatry, 145, 339.

Bhutani GE, Montaldi D, Brooks DN and McCulloch J (1992) A neuropsychological investigation into frontal lobe involvement in dementia of the Alzheimer type. Neuropsychology, 6, 211-224.

Bondareff W, Mountjoy CQ and Roth M (1982) Loss of neurons of origin of the adrenergic projection to cerebral cortex (nucleus locus coeruleus) in senile dementia. Neurology, 32, 164-168.

Bowen DM, Najlerahim A, Proctor AW, Francis PT and Murphy E (1989) Circumscribed changes in the cerebral cortex in neuropsychiatric disorders of later life. Proceedings of the National Academy of Sciences of the USA, 86, 9540-9508.

Braak $H$ and Braak E (1991) Neuropsychological staging of Alzheimer-related changes. Acta Neuropathologica, 82, 239-251

Brady DR and Mufson EJ (1990) Amygdaloid pathology in Alzheimer's disease, qualitative and quantitative analysis. Dementia, 1, 5-17.

Broks P, Preston GC, Traub M, Poppleton P, Ward C and Stahl SM (1988) Modelling dementia: effects of scopolamine on memory and attention. Neuropsychologia, 26, $685-700$.

Brun A (1987) Frontal lobe degeneration of non-Alzheimer type: I. Neuropathology. Archives of Gerontology and Geriatrics, 6, 193-208.

Brun A, Englund B, Gustafson L, Passant U, Mann DMA, Neary D and Snowden JS (1994) Clinical and neuropathological criteria for fronto-temporal dementia. Journal of Neurology, Neurosurgery and Psychiatry, 57, 416-418.

Christensen A-L (1974) Luria's Neuropsychological Investigation. Munksgaard, Copenhagen.

DeLacoste M-C and White D (1993) The role of cortical connectivity in Alzheimer's disease pathogenesis: a review and model system. Neurobiology of Aging, 14, $1-16$.

DeRenzi E and Faglioni P (1978) Normative data and screening power of a shortened version of the token test. Cortex, 14, 41-49.

Fischer P, Marterer A and Danielczyk W (1990) Right-left disorientation in dementia of the Alzheimer type. Neurology, 40, 1619-1620.

Folstein MF, Folstein SW and McHugh PR (1975) "Mini Mental State": a practical method of grading the cognitive state of patients for the clinician. Journal of Psychiatric Research, 12, 189-198.

Freedman M, Leach L, Kaplan E, Winocur G, Shulman KI, and Delis DC (1994) Clock drawing: A Neuropsychological analysis. Oxford University Press.

Grady CL, Haxby JV, Horwitz D, Sundaram M, Berg G, Schapiro M, Friedland RP and Rapoport SI (1988) Longitudinal study of the neuropsychological and cerebral metabolic changes in dementia of the Alzheimer type. Journal of Clinical and Experimental Neuropsychology, 10, 576-596.

Haxby JV and Rapoport SI (1986) Abnormalities of regional brain metabolism in Alzheimer's disease and their relation to functional impairment. Progress in Neuropsychopharmacology and Biological Psychiatry, 10, 427-438.
Haxby JV, Grady CL, Duara R, Schlageter N, Berg G and Rapoport SI (1986) Neocortical metabolic abnormalities precede non memory cognitive deficits in early Alzheimer's-type dementia. Archives of Neurology, 43, 882-885.

Herzog AB and Kemper TL (1980) Amygdaloid changes in ageing and dementia. Archives of Neurology, 37, 625-629.

Holman BL (1986) Perfusion and receptor SPECT in the dementias. George Taplin memorial lecture. Journal of Nuclear Medicine, 27, 855-860.

Huppert FA and Tym E (1986) Clinical and neuropsychological assessment of dementia. British Medical Bulletin, 42, 11-18.

Iversen LL, Rossor MN and Reynolds GP et al (1983) Loss of pigmented dopamine-beta-hydroxylase positive cells from locus coeruleus in senile dementia of the Alzheimer's type. Neuroscience Letters, 39, 95-100.

Jagust WJ, Budinger TF and Reed BR (1987) The diagnosis of dementia with single photon emission computed tomography. Archives of Neurology, 44, 258-262.

Johnson KA, Holman BL, Mueller SP, Rosen TJ, English R, Nagel JS and Growden JH (1988) Single photon emission computed tomography in Alzheimer's disease: abnormal iofetamine I-123 uptake reflects dementia severity. Archives of Neurology, 45, 392-396.

Kessler J, Herholz I, Slansky R, Mielke R, Grond M and Heiss WD (1993) Does impairment of frontal lobe tasks reflect frontal lobe degeneration in patients with probable Alzheimer's disease? Journal of Cerebral Blood Flow and Metabolism, 13, S236.

Kumar A, Schapiro MB, Grady C, Haxby JB, Wagner G, Salerno JA, Friedland RP and Rapoport SI (1991) High resolution PET studies in Alzheimer's disease. Neuropsychopharmacology, 4, 35-46.

Lines CR, Dawson C, Preston GC, Reich S, Foster C and Traub M (1991) Memory and attention in patients with senile dementia of the Alzheimer type and in normal elderly subjects. Journal of Clinical and Experimental Neuropsychology, 13, 691-702.

Mann DMA (1992) The neuropathology of the amygdala in ageing and dementia. In: The Amygdala: Neurobiological Aspects of Emotion, Memory, and Mental Dysfunction (Ed. JP Aggleton), Wiley, New York pp. 575-593.

Mann DMA, Yates PO and Marcyniuk B (1985) Some morphometric observations on the cerebral cortex and hippocampus in presenile Alzheimer's disease, Senile Dementia of the Alzheimer Type and Down's Syndrome. Journal of Neurological Sciences, 69, 139-159.

Maunsell JHR and Newsome WT (1987) Visual processing in monkey extrastriate cortex. Annual Review of Neuroscience, 10, 363-401.

McCarthy RA and Warrington EK (1990) Cognitive Neuropsychology: A Clinical Introduction. Academic Press, San Diego.

McKhann G, Drachman D, Folstein M, Katzman R, Price $\mathrm{D}$ and Stadlan EM (1984) Clinical diagnosis of Alzheimer's disease: report of the NINCDS-ADRDA work group under the auspices of the Department of Health and Human Services task force on Alzheimer's disease. Neurology, 34, 939-944. 
Montaldi D, Brooks DN, McColl JH, Wyper D, Patterson J, Barron E and McCulloch J (1990) Measurements of rCBF and cognitive performance in Alzheimer's disease. Journal of Neurology, Neurosurgery and Psychiatry, 53, 33-38.

Moore V and Wyke MA (1984) Drawing disability in patients with senile dementia. Psychological Medicine, 14, 97-105.

Moss MB and Albert MS (1988) Alzheimer's disease and other dementing disorders. In: Geriatric Neuropsychology (Eds MS Albert and MB Moss), Guilford Press, New York.

Najlerahim A and Bowen DM (1988) Regional weight loss of the cerebral cortex and some subcortical nuclei in senile dementia of the Alzheimer type. Acta Neuropsychologica, 75, 509-512.

Neary D, Snowden JS, Northen B and Goulding P (1988) Dementia of frontal lobe type. Journal of Neurology, Neurosurgery and Psychiatry, 51, 353-361.

Nelson HE and Willison J (1991) National Adult reading Test (2nd Edition). NFER-NELSON, Windsor.

Newcombe F and Russell WR (1969) Dissociated visual perceptual and spatial deficits in focal lesions of the right hemisphere. Journal of Neurology, Neurosurgery and Psychiatry, 32, 73-81.

Newcombe F, Ratcliff G and Damasio H (1987) Dissociable visual and spatial impairments following right posterior cerebral lesions: clinical, neuropsychological and anatomical evidence. Neuropsychologia, 25, 149-161.

Owen AM, James M, Leish PN, Summers BA, Mardsen CD, Quinn NP, Lange KW and Robbins TW (1992) Fronto-striatal cognitive deficits at different stages of Parksinson's disease. Brain, 115, 1727-1751.

Pappata S, Mazoyer B, Tran Dinh S, Cambon H, Levasseur M and Baron JC (1990) Effects of capsular or thalamic stroke on metabolism in the cortex and cerebellum: a positron emission tomography study. Stroke, 21, 519-524.

Pillon B, Dubois B, L'Hermitte F and Agid F (1986) Heterogeneity of cognitive impairment in Progressive Supranuclear Palsy, Parkinson's disease, and Alzheimer's disease. Neurology, 36, 1179-1185.

Rapoport SI (1991) Positron emission tomography in Alzheimer's disease in relation to disease pathogenesis: a critical review. Cerebrovascular and Brain Metabolism Reviews, 3, 297-335.

Reige WH and Metter EJ (1986) Cognitive and brain imaging measures of Alzheimer's disease. Neurobiology of Aging, 9, 69-86.

Sahakian BJ, Downes JJ, Eagger, S, Evenden JL, Levy L, Philpot MP, Roberts AC and Robbins TW (1990) Sparing of attentional relative to mnemonic function in a subgroup of patients with dementia of the Alzheimer type. Neuropsychologia, 28, 1197-1213.

Samson Y, Baron JC, Bousser MG, Rey A, Derlon JM, David P and Comoy J (1985) Stroke, 16, 606-616.

Saper CB (1988) Chemical neuroanatomy of Alzheimer's disease. In: Handbook of Psychopharmacology Volume 20. Eds LL Iversen, SD Iversen and SH Snyder. Plenum Press, New York.
Shallice T (1982) Specific impairments of planning. Philosophical Transactions of the Royal Society of London, Series B, 298, 199-209.

Shallice T (1988) Neuropsychology to Mental Structure. Cambridge University Press, Cambridge.

Shallice T and Evans ME (1978) The involvement of the frontal lobes in cognitive estimation. Cortex, 14, 294-303.

Shuttleworth EC (1984) Atypical presentations of dementia of the Alzheimer type. Journal of the American Geriatric Society, 32, 485-490.

Soh K, Larsen B, Skinhoj E and Lassen NA (1978) Regional cerebral blood flow in aphasia. Archives of Neurology, 35, 625-632.

Terry RD, Peck A, Detersa R, Schechter R and Horoupian DS (1981) Some morphometric aspects of the brain in senile dementia of the Alzheimer type. Annals of Neurology, 10, 184-192.

Tomlinson BE, Irving D and Blessed G (1981) Cell loss in the locus coeruleus in senile dementia of the Alzheimer type. Journal of Neurological Science, 49, 419-429.

Tranel D and Hyman BT (1990) Neuropsychological correlates of bilateral amygdala damage. Archives of Neurology, 47, 349-355.

Trenerry MR, Crosson B, DeBoe J and Leber WR (1989) Stroop Neuropsychological Screening Test. Psychological Assessment Resources, Inc., Odessa, Florida.

Ungerleider LG and Mishkin M (1982) Two cortical visual systems. In: The Analysis of Visual Behaviour Eds DJ Ingle, $\mathrm{MH}$ Goodale and RJW Mansfield. MIT Press, Cambridge, MA pp. 549-586.

Ungerleider LG and Haxby JV (1994) 'What' and 'where' in the human brain. Current Opinion in Neurobiology, 4 , $157-165$

Von Monakow CV (1911) von lokalization der hirnfunktionen. Journal fur Psychologie und Neurologie, 17, 185-200, 1911. (Reprinted in G von Bonin, The Cerebral Cortex. Charles C Thomas, 1960, Springfield, IL).

Warrington EK and James M (1967) Disorders of visual perception in patients with localised cerebral lesions. Neuropsychologia , 5, 253-266.

Warrington EK and James M (1988) Visual apperceptive agnosia: a clinico-anatomical study of three cases. Cortex, 24, 13-32.

Warrington EK and James M (1991) The Visual Object and Space Perception Battery. Thames Valley Test Company, Bury St Edmunds.

Whitehouse PJ, Price DL, Clark AW, Coyle JT and DeLong MR (1981) Alzheimer's disease: evidence for selective loss of cholinergic neurons in the nucleus basalis. Annals of Neurology, 10, 122-126.

Wechsler D (1981) Wechsler Adult Intelligence Scale Revised. The Psychological Corporation/Harcourt Brace Jovanovich, New York.

Wilcock GK, Esiri MM, Bowen DM and Hughes AO (1988) The differential involvement of subcortical nuclei in senile dementia of the Alzheimer's type. Journal of Neurology, Neurosurgery and Psychiatry, 51, 842-849.

Wilkins AJ, Shallice T and McCarthy R (1987) Frontal lesions and sustained attention. Neuropsychologia, 25, 359-365. 


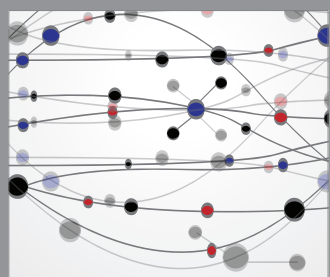

The Scientific World Journal
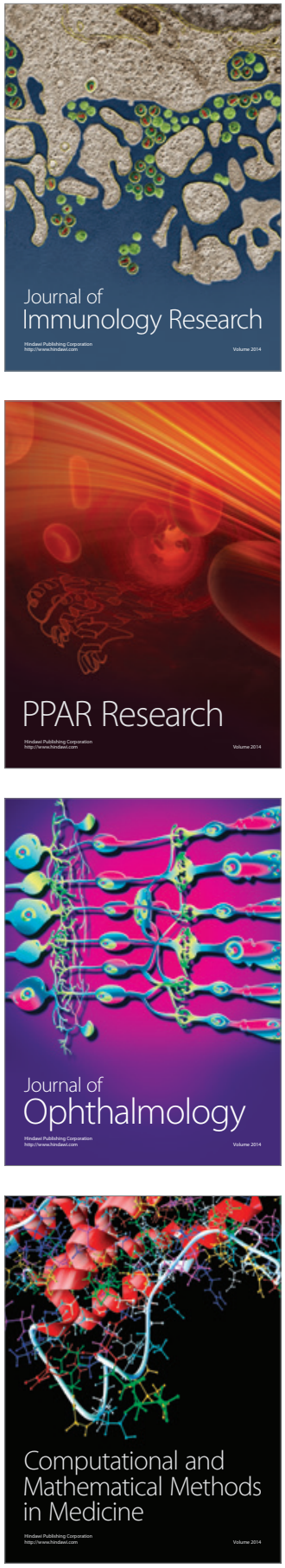

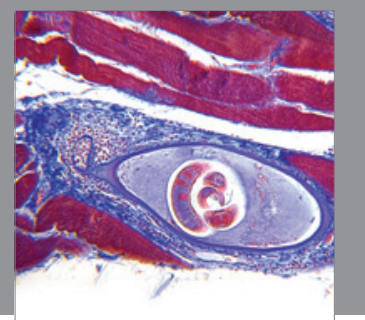

Gastroenterology

Research and Practice
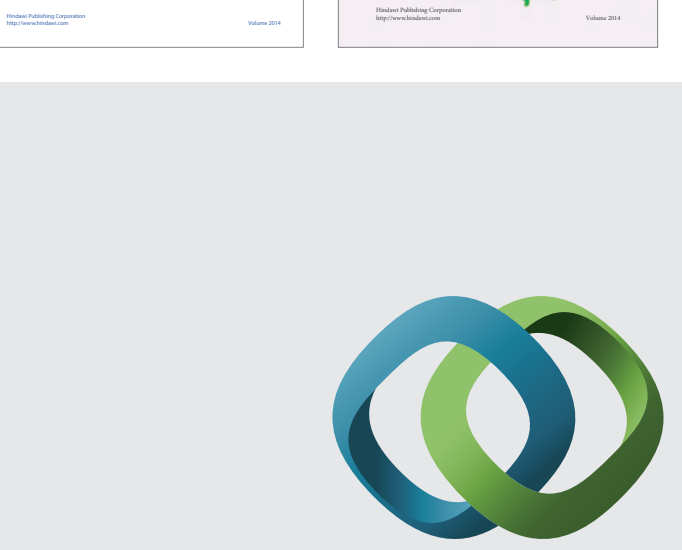

\section{Hindawi}

Submit your manuscripts at

http://www.hindawi.com
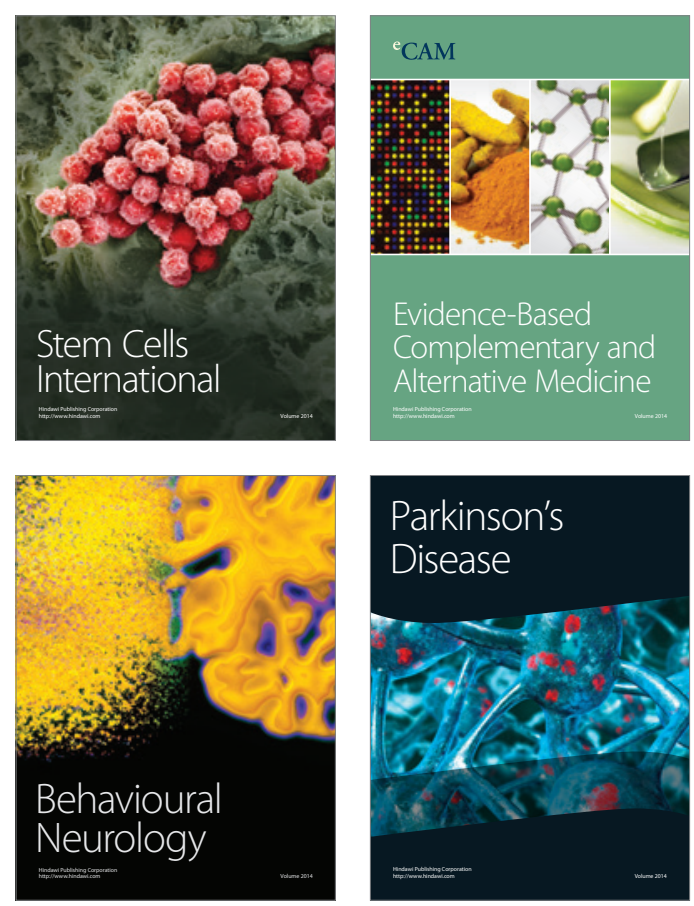

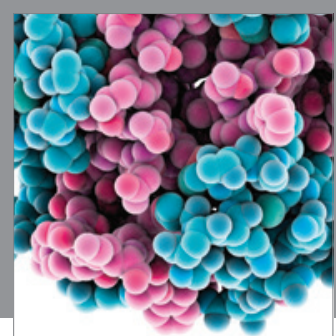

Journal of
Diabetes Research

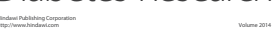

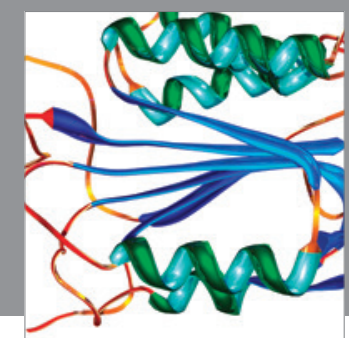

Disease Markers
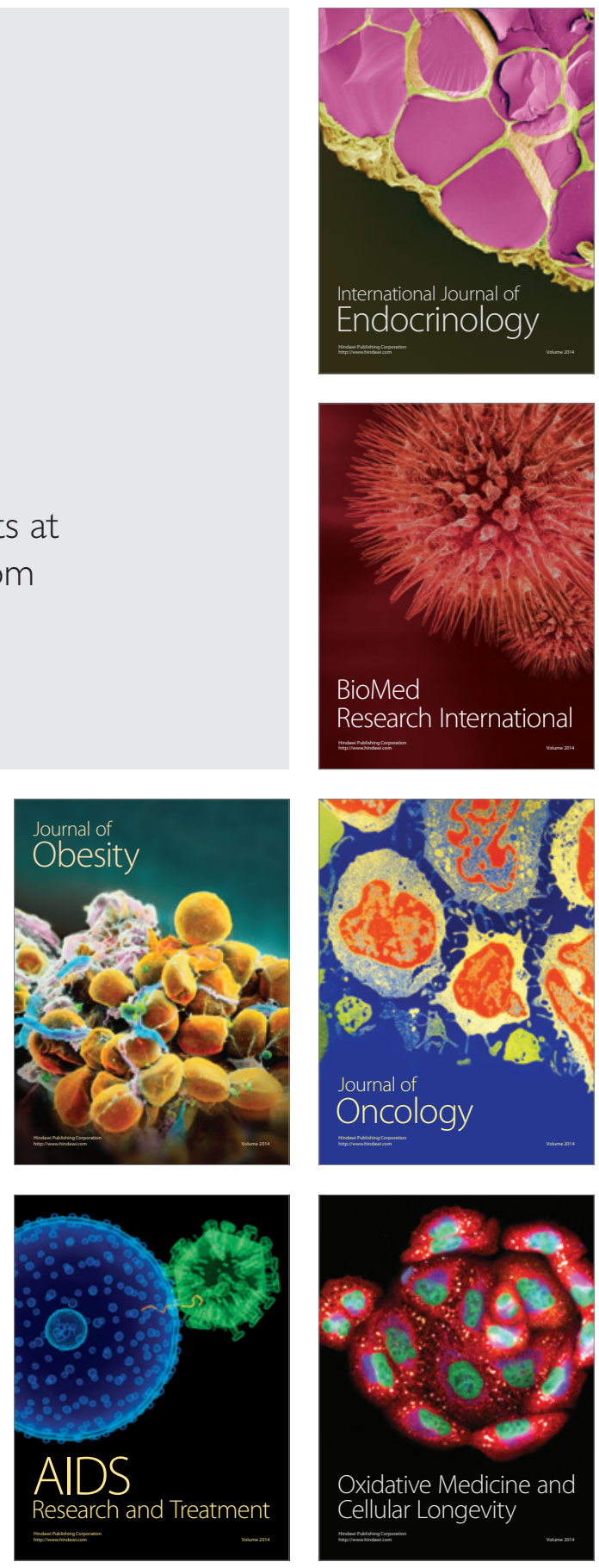